\title{
Improving memory for color
}

\author{
MICHAEL H. SIEGEL and DAVID E. SIEGEL \\ State University of New York, Oneonta, New York 13820
}

\begin{abstract}
One problem with remembering color is the absence of a precise verbal code. An experiment was performed in which one group taught such a code was more accurate and more consistent than a second group which was not taught this code.
\end{abstract}

Perception of visual stimuli is changed over time (Sperling, 1960). Woodworth and Schlosberg (1954) have discussed the concept of memory color as a device to enhance color consistency. A more recent review of the experimental literature of the influence of memory on perception was conducted by Tate and Springer (1971). Objects are remembered with the colors we originally learned. For identifiable objects, perception of color may be partially a function of information coded in memory.

Although most memory studies involving visual perception have dealt with easily codable stimuli, there have been exceptions. Hanawalt and Post (1942) tested Koffka's notion that the memory trace for color changes in the direction of a more saturated color. Their results failed to support this notion.

Bartlett (1932) in his now classic text, Remembering, has suggested that naming a stimulus array assists memory. Indeed, the recent revitalization of imagery research makes the identical point. Broadbent (1958) and others have extended this view.

In a typical experimental situation concerned with color memory, a subject is called upon to select or to match a color he has seen some time ago. The task is different from other memory problems involving words or other symbols, possibly because of the relatively impoverished vocabulary of most normal subjects as compared with the richness of their color experiences. That is, most subjects find it impossible to employ a precise verbal label for a color experience. Our laboratory has been working on a perceptual color scale in which numbers are assigned to a stimulus to represent its color appearance (Siegel \& Siegel, 1971). Thus, ill-defined color names may be replaced by highly defined color numbers. The present study provided a situation in which the usual color names could be extended and thereby permit subjects an opportunity to code their color experiences symbolically. The question to be answered is simply, does the presence of such a coding system permit greater precision of color memory?

\section{METHOD}

\section{Subjects}

Subjects for the study were 12 volunteers. On the basis of chance, six were assigned to the experimental condition and the remaining six to the control condition. All subjects had normal color vision, but none had previously served in any vision experiments; indeed, none were familiar with the problem or the apparatus prior to experimentation.

\section{Apparatus}

A circular 3 deg stimulus field was produced by imaging light from the exit slit of a 500-mm focal length Bausch and Lomb monochromator into the observer's right eye. The field was in Maxwellian view and was observed through a 3-mm artificial pupil. Stimulus luminance was maintained at $1 \mathrm{fL}$ by means of a series of neutral density filters. A wavelength calibration performed both before and after the study revealed no shift in nominal values of wavelength settings throughout the study.

\section{Procedure}

The procedure employed was identical for all subjects except for the contents of an instructional session prior to experimentation. Subjects in the control group were presented a lecture on the relationship of hue to wavelength for $10 \mathrm{~min}$. Subjects in the experimental group instead were given a 10-min lecture describing a coding scheme by which numbers could be assigned to hue experiences. First, subjects were told that their hue experiences could be represented by a diamond such as that indicated in Figure 1. Psychologically distinct hues may be placed at each corner. The color names blue, green, yellow and red were replaced by numbers $5,15,25$ and 35 , and that mixture of blue and red exactly intermediate was called 0 or 40 , as in Figure 2. With this numbering scheme, it is possible to extend the usable color names to 40 whole numbers. Thus, a hue experience of blue-green could be assigned a number between 5 and 15, the exact number dependent upon the subject's judgment of relative greenness or blueness.

The task for each subject consisted of matching hues from memory. Subjects were seated in a light-controlled cubicle and

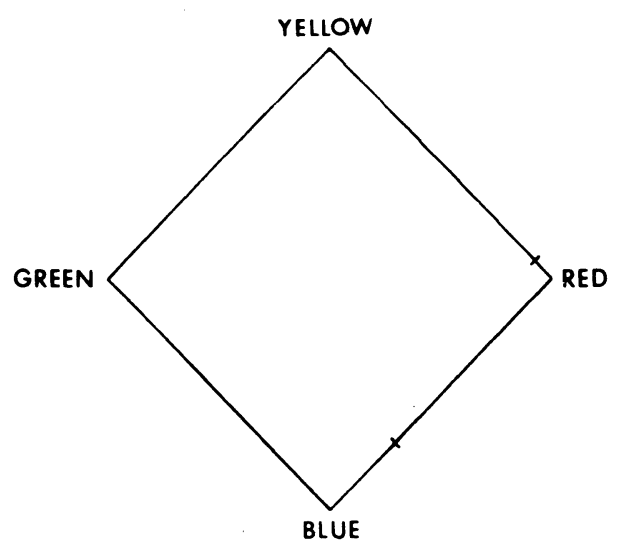

Figure 1. Hue diamond representing four psychologically distinct hues. 


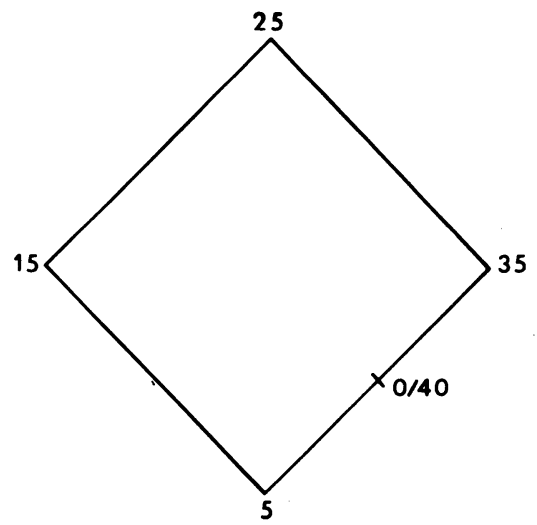

Figure 2. Numbering code in which numbers from $\mathbf{0}$ to $\mathbf{4 0}$ are used to represent color names.

observed an aperture in a white screen through which the stimulus appeared. Observation was with a $3-\mathrm{mm}$ artificial pupil. Any of 14 different wavelengths from $420 \mathrm{~nm}$ to $640 \mathrm{~nm}$ could be presented for $5 \mathrm{sec}$ as a trial. After the stimulus was observed, the field was darkened for $15 \mathrm{sec}$, during which time the experimenter could change the wavelength. When the field reappeared, the test stimulus was set to one of five wavelength values relative to the original stimulus, either $+10-,+5-,-10-$, $-5-$, or $0-\mathrm{nm}$ difference. Each subject received two trials at each wavelength setting, making a total of 140 trials. The subject's task was to direct the experimenter to adjust the wavelength of the test stimulus until it matched the original stimulus. Although subjects were allowed to bracket a wavelength, most tended to have the wavelength drum moved consistently in a single direction until a match point had been reached.

\section{RESULTS AND DISCUSSION}

Initially, results for both experimental and control groups were evaluated by plotting the mean wavelength set by subjects in each of these conditions at each of the 14 wavelength values used in the study. Figure 3 presents these results. Even a brief inspection of these results shows that subjects appear to be incredibly accurate. Indeed, the line relating the matched wavelength to the test wavelength is indistinguishable from one showing perfect correspondence. Second, it seems obvious that there are no differences in accuracy between the control and experimental subjects. These conclusions are confirmed by an analysis of variance. We are left, then, with the strange conclusion that color setting accuracy remains perfect even over a $15-\mathrm{sec}$ period and, perhaps even stranger, that the group with additional color categories is no more accurate than the group with no such extra categories.

When the standard deviations of subjects' settings are calculated as in Figure 4, these conclusions are seen to be premature and questionable. Clearly, the control data are far more variable than the experimental. Again this is confirmed by $\mathrm{F}$ tests.

There is no inconsistency between data in Figures 3 and 4 . Neither the experimental nor control group is perfect in matching. The apparent accuracy results from averaging mistakes which appear symmetrically around each wavelength. What is required is some method of scoring which does not permit cancellation of positive
Figure 3. Mean wavelength set in nanometer as a function of wavelength in nanometers.

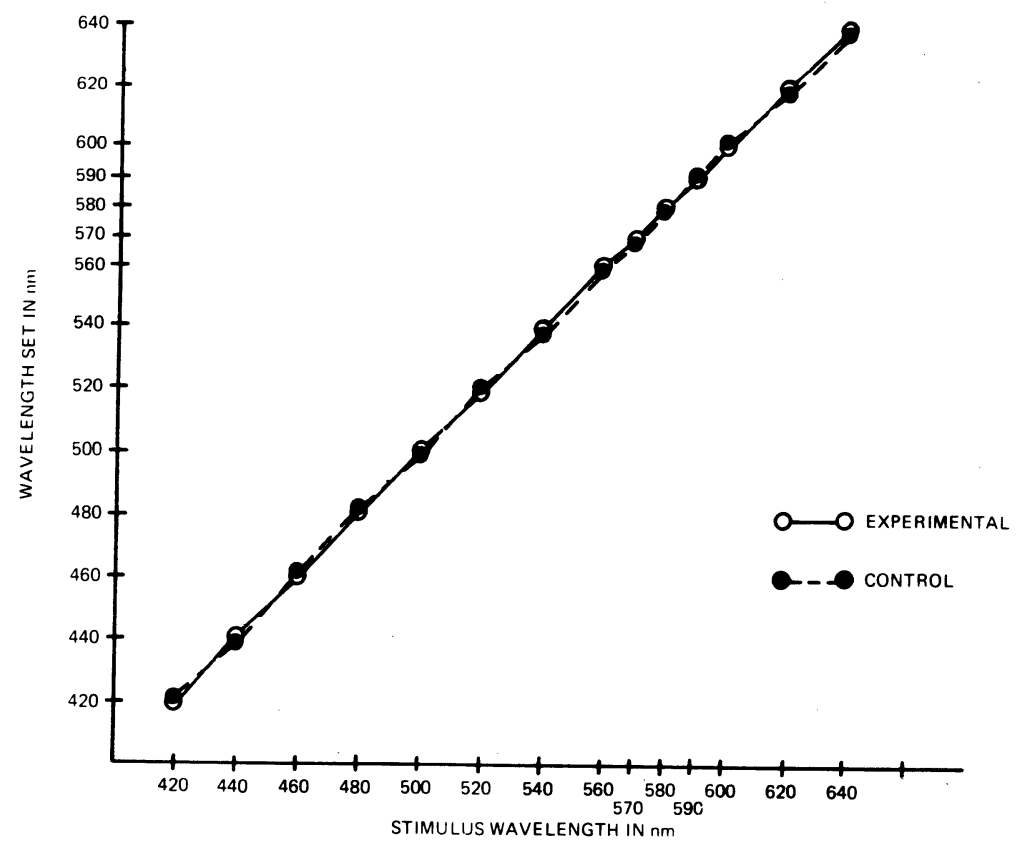




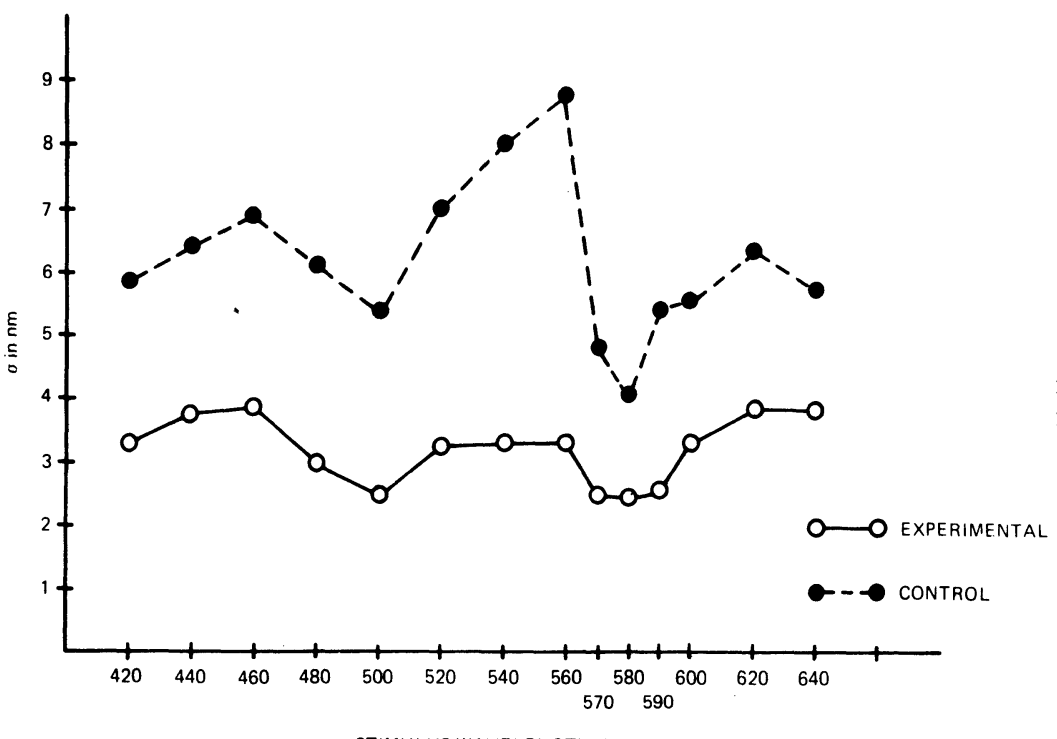

Figure 4. Standard deviations in nanometer as a function of wavelength in nanometers.

and negative errors. We decided to plot the absolute error which fulfills this requirement. The results of plotting mean absolute error at each wavelength for the experimental and control groups are given in Figure 5. Not only are the experimental subjects less variable (see Figure 4), but they are also far more accurate than control subjects. An analysis of variance shows that the curves differed significantly at each of the 14 wavelengths studied.

Figure 6 shows the relation between accuracy as defined by absolute error and wavelength setting differences across all wavelengths studied. For both the control and the experimental groups, when there was 0 -nm difference between the original stimulus and the test stimulus, errors were consistently less. The other values showed no reliable pattern. It should be noted, however, that no subject was aware that the original and test stimuli were ever equal.

When Figure 5 is re-examined it seems reminiscent of the shape of the wavelength discrimination curve. Figure 7 shows results of a wavelength discrimination study conducted some time ago (Siegel, 1964). The points of correspondence or similarity are the regions of greatest accuracy in the spectral regions, blue and yellow. While matching hues from memory is a different task from discriminating between two hues, there is clearly a relationship. We could expect the similarity between the curves to disappear as the interval between original and test stimuli becomes increased, at least for subjects without a special code.

In summary, the results clearly demonstrate that those subjects who are provided with additional verbal categories for color used them and could match hues with surprising accuracy. Normal language does not pro-

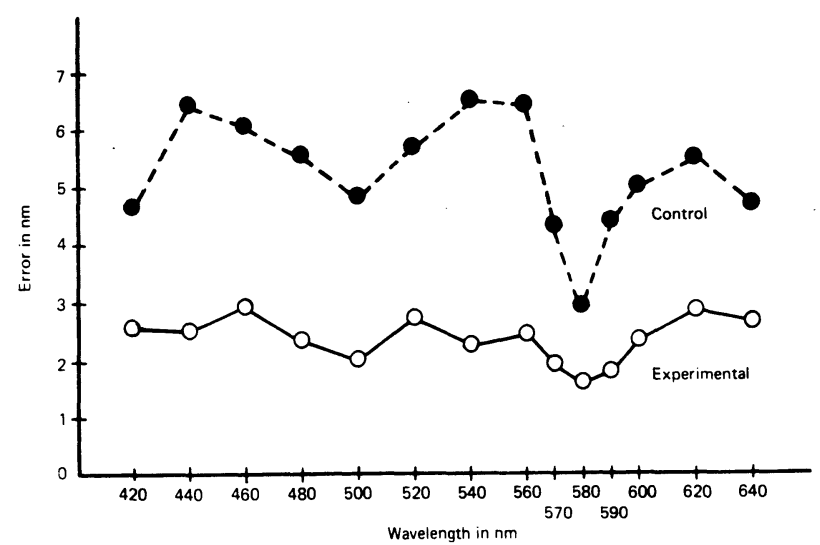

Figure 5. Mean absolute error in nanometer as a function of wavelength in nanometer.

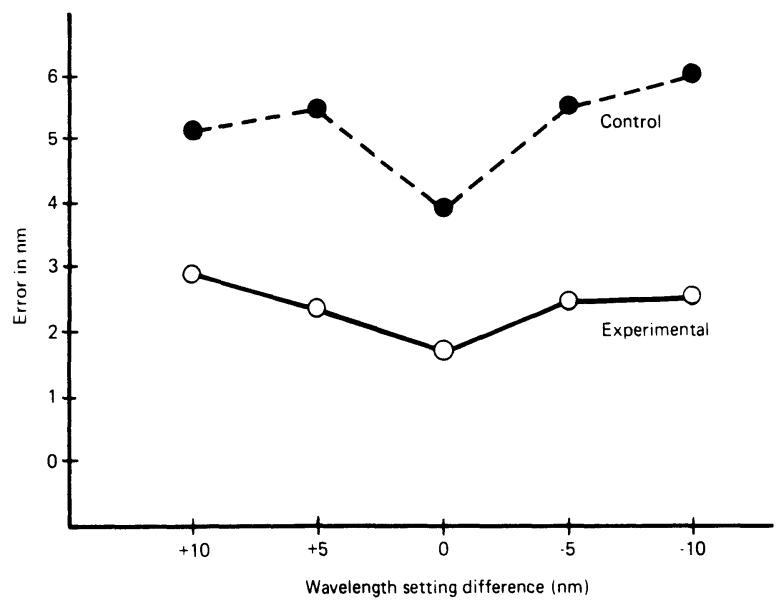

Figure 6. Mean absolute error in nanometer as a function of wavelength setting difference in nanometers. 


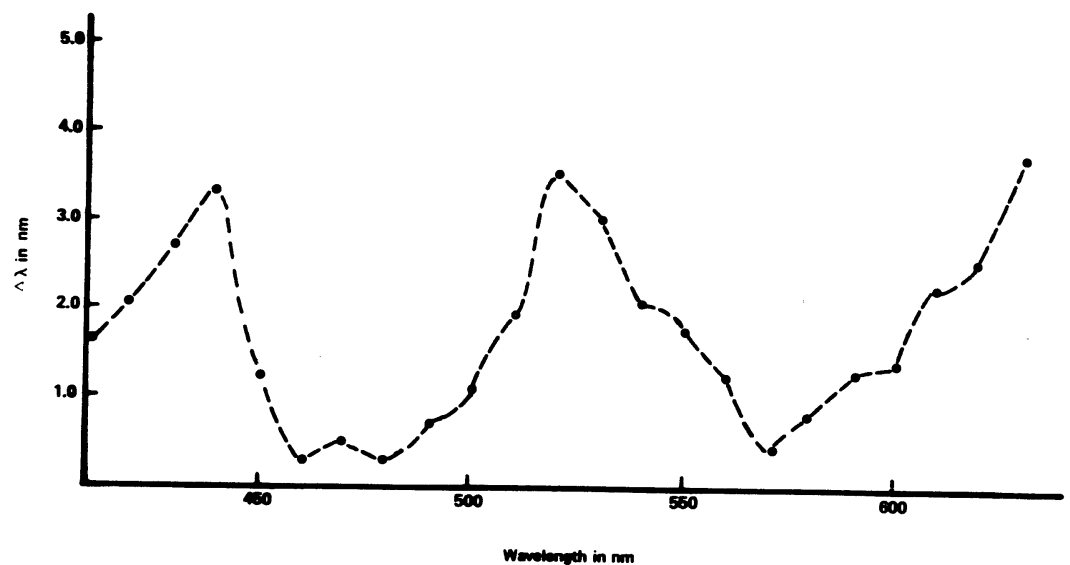

Figure 7. Wavelength discrimination curve from earlier study (see text).

vide very many categories for fine judgments of hue. The ability to remember a hue is enhanced by a coding process which provides additional categories.

\section{REFERENCES}

Bartlett. F. C. Remembering: A study in experimental and social psichology: New York: MacMillan, 1932.

Broadbent. D. E. Perception and communication. New York: MacMillian (Pergamon). 1958.

Ha.iawalt. N. G..d Post. B. E. Memory trace for color. Journal of Experimental Psychology. 1942. 30. 216-227.

SIfGel, M. H. Discrimination of Color: IV. Sensitivity as a function of spectral wavelength, 410 through $500 \mathrm{~nm}$. Journal of the Optical Society of America. 1964, 54. 821-823.

Siegel. M. H.. \& Siegel. A. B. A comparison of techniques for measuring hue appearance. Behavior Research Methods \& Instrumentation, 1971, 3, 1-4.

SPerling, G. The information available in brief visual presentations. Psychological Monographs. 1960. 74. No. 11 (Whole No. 498).

Tate. J. D. \& Springer. R. M. Effects of memory time in successive judgments. Psychological Bulletin. 1971, 76. 344-4()8.

Woodworth. R. S. \& Schlosberg, H. Experimental Psychology: (Rev. ed.) New York: Holt, 1954.

( Received for publication February 19. 1976.) 